\title{
Why We Need a Testbed for Black-Box Optimization Algorithms in Building Simulation
}

\author{
Christoph Waibel $^{1,2}$, Thomas Wortmann ${ }^{3}$, Georgios Mavromatidis ${ }^{1,2}$, \\ Ralph Evins ${ }^{4}$, Jan Carmeliet ${ }^{1}$ \\ ${ }^{1}$ Chair of Building Physics, ETH Zurich, Switzerland \\ ${ }^{2}$ Urban Energy Systems Laboratory, Empa, Dübendorf, Switzerland \\ ${ }^{3}$ Department of Architecture, Xi'an Jiaotong Liverpool University, Suzhou, China \\ ${ }^{4}$ Energy Systems and Sustainable Cities, University of Victoria, Canada
}

\begin{abstract}
When applying black-box optimization (BBO) algorithms there seems to be a lack of guidelines on which algorithm to select and how to properly tune their algorithmic parameters. Many benchmarks are conducted either on large sets of mathematical test functions or on few building simulation problems. This inhibits us from drawing generalizable conclusions valid over the entire domain of building energy optimization (BEO). As a consequence, we argue that $\mathrm{BEO}$ urgently needs a unified testbed for consistently benchmarking and researching BBO algorithms. We illustrate our point by conducting a Fitness Landscape Analysis (FLA) of several building simulation problems using EnergyPlus, a solar potential simulator and Fast Fluid Dynamics, and comparing it to common mathematical test functions. For a number of FLA metrics we can demonstrate that building simulation problems differ significantly. Furthermore, by benchmarking a number of BBO algorithms on a BEO and test function set separately, we show that algorithm performance depends on the problem set, thus leading to the conclusion that the domain of building simulation requires a dedicated testbed to facilitate the application of black-box optimization.
\end{abstract}

\section{Introduction}

Black-box optimization (BBO) algorithms (also: derivative-free optimization, search heuristics or metaheuristics) (cp. Audet and Hare, 2017) are gaining popularity in both research and practice, but the current research and knowledge gap includes a lack of general guidelines on which algorithm to select and how to properly tune their algorithmic parameters (Evins, 2013; Nguyen et al., 2014). Several studies try to answer these questions by benchmarking algorithms on either mathematical test functions (Rios and Sahinidis, 2013), or on only few building simulation problems, which are also different from publication to publication (Wetter and Wright, 2004; Kämpf et al., 2010; Hamdy et al., 2016). This inhibits us from drawing generalizable conclusions valid over the entire domain of simulation-based building optimiza- tion. As a consequence, we argue that building simulation urgently needs a unified testbed for consistently benchmarking and researching BBO algorithms.

The BBO community conducts annual benchmark competitions on a large set of mathematical test functions and also recently introduced some realworld problems from different domains (mechanical and civil engineering, computer-science) (e.g. COCO and BBComp $)^{1,2}$. An important factor in real-world problems are limited available resources for solving a problem induced by evaluating a design proposaleither using a time-expensive simulator or costly real experiments - resulting in a relatively small evaluation budget. This contrasts to the large evaluation budgets used in the mathematical test functions, which are unfeasible in real-world problems when the function evaluation is expensive. Furthermore, we state the hypothesis that building simulation problems exhibit features that can be used to discriminate them from other domains and especially from mathematical test functions. Thus, it would be desirable to undertake domain-specific BBO research to better match requirements present in building simulation.

We corroborate our point in this paper by conducting a Fitness Landscape Analysis (FLA) of several building simulation problems using EnergyPlus, a solar potential simulator and Fast Fluid Dynamics and comparing it with mathematical test functions. The FLA provides us with various metrics showing the difference between simulation-based building problems and test functions, thus leading to the rationale of establishing a specialized building simulation testbed. Previous studies indicate discontinuities and highly multi-modal cost landscapes in building energy optimization problems (Wetter and Wright, 2004; Wortmann et al., 2017), but generalizable findings have yet to be formulated.

In the following sections, we address the question whether building simulation problems can be discriminated from mathematical test functions. The novelty is a quantified comparison of simulation-based

\footnotetext{
${ }^{1}$ http://coco.gforge.inria.fr/

${ }^{2}$ https://bbcomp.ini.rub.de/
} 
building design problems and mathematical test functions using techniques from FLA, demonstrating how such problems differ from each other. Furthermore, we propose to establish a problem testbed related to building simulation (energy demand, building systems, solar, CFD, etc.), which should facilitate a transparent and systematic benchmarking of algorithms. We demonstrate the benefits of such a testbed with empirical numerical experiments and problem analysis.

\section{Optimization problems considered}

We consider commonly used mathematical test functions and building energy optimization problems using EnergyPlus (Crawley et al., 2000) 8.5.0, a solar potential simulator (Waibel et al., 2017) and a Fast Fluid Dynamics program (Waibel et al., 2017). All problems are implemented using Rhinoceros 3D Grasshopper (Rhino) as simulation platform. Each problem is described briefly in the following sections; they can be accessed online for reproducibility ${ }^{3}$.

The building energy problems have also been used in a recent benchmark on global search algorithms (Waibel et al., 2019). The present conference paper provides additional content by presenting a Fitness Landscape Analysis and quantitatively comparing the simulation-based problems with mathematical test functions. Also, the solar and airflow problems were not included in Waibel et al. (2019).

\section{Mathematical test functions}

We include 5 mathematical test functions, found in Kämpf et al. (2010), who conducted a benchmark of their custom BBO algorithm on these test functions and 2 building energy optimization problems. The test function problems are denoted as $p_{1}^{\mathrm{tf}}$ and $p_{5}^{\mathrm{tf}}$ ("Sphere" in $n=10$ and $20, n$ being the problem dimension), $p_{2}^{\mathrm{tf}}$ and $p_{6}^{\mathrm{tf}}$ ("Ackley" in $n=10$ and $20), p_{3}^{\mathrm{tf}}$ and $p_{7}^{\mathrm{tf}}$ ("Rastrigin" in $n=10$ and 20), $p_{4}^{\mathrm{tf}}$ and $p_{8}^{\mathrm{tf}}$ ("Rosenbrock" in $n=10$ and 20), and $p_{9}^{\mathrm{tf}}$ ("Constrained" in $n=13$ ). The functions are given in Kämpf et al. (2010).

For the benchmark presented later we shift the domains of the test functions slightly, since the algorithm DIRECT would otherwise identify the global optimum (which for most test functions lies at $x_{i}=$ $0, \forall i$ ) at the first function evaluation ${ }^{4}$.

\section{Building energy problems}

We include 15 building energy optimization problems from the literature $(n=4$ to $n=35)$, denoted with $p_{1}^{\text {beo }}$ to $p_{15}^{\text {beo }}$. All use EnergyPlus as simulator, but they use different variable types (geometry, construction types, control set points, etc.) and cost functions. Originally, the building energy problems have been published in Wetter and Wright (2004); Djuric

\footnotetext{
${ }^{3}$ https://github.com/christophwaibel/BEOBenchmark

${ }^{4}$ DIRECT's deterministic procedure starts its search at the domain center.
}

et al. (2007); Kämpf et al. (2010); Nguyen and Reiter (2014); Ramallo-González and Coley (2014); Waibel et al. (2016).

\section{Solar problems}

We include 2 solar optimization problems $p_{1}^{\text {sol }}$ and $p_{2}^{\text {sol }}$ that are reproduced based on Kämpf and Robinson (2010) and Yi and Kim (2015). One problem $(n=31)$ concerns the shape of building roofs in Switzerland by altering the height of surface vertices with the objective to maximize total annual solar potentials in $\mathrm{kWh} / \mathrm{m}^{2} \mathrm{a}$; the other problem $(n=8)$ concerns the rotation and twist of residential high-rise buildings in China with the objective to maximize days in the year with at least 2 hours of direct solar access on the building façades.

\section{Airflow problems}

We include 2 airflow optimization problems $-p_{1}^{\text {wnd }}$ and $p_{2}^{\text {wnd }}$-in this paper. Both problems assume a prevailing wind direction as a simplification. $p_{1}^{\text {wnd }}$, inspired by Wilkinson and Hanna (2014), concerns wind load minimization of a high-rise building. The two decision variables are the rotation of the bottom and the top floorplan, which can twist the structure. We develop a second wind problem with 12 decision variables for rotating and positioning 4 cubic buildings. The objective is to maximize the natural ventilation potential of all buildings.

\section{Fitness Landscape Analysis}

Fitness Landscape Analysis (FLA) deals with the study of the input-output relations of optimization problems (Pitzer and Affenzeller, 2012). The word "Fitness" is synonymous with the objective function value and "Landscape" refers to the multivariate costresponse $f(\boldsymbol{x})$. In 2-dimensional problems, a visualization is straightforward, as $f(\boldsymbol{x})$ can be plotted on the Z-axis. Many problems however have $n>2$ and therefore techniques and metrics from FLA can be utilized to visualize and quantify problem structure. FLA can be distinguished between "static" and "dynamic". The latter concerns the relation between problem difficulty and a specific optimization algorithm trying to solve this particular problem, whereas static FLA is independent of the optimizer. As we are interested in finding discriminating features between test functions and building simulation problems, we conduct a static FLA in this paper.

As FLA metrics we report Morris indices (a screening global sensitivity analysis technique), fitness distance correlation (FDC) that correlates the objective function (or cost) values with the distance of solutions in variable space, and autocorrelation factors (ACF). Generally, the specific units of the cost functions are irrelevant (e.g. $\mathrm{kWh}, \$$, etc.) in our FLA, since we normalize them such that we can use unified metrics. Therefore, we omit the units in the following plots and discussions. 


\section{Fitness distance correlation}

Fitness distance correlation has been introduced by Jones and Forrest (1995) and reduces a multivariate space into a single distance metric $d$, therefore allowing to compute the correlation between $d$ and $f(\boldsymbol{x})$. A large FDC value therefore shows a high correlation between decision variables and the cost value. Consequently, low FDC values may indicate higher problem difficulty, as there is more ambiguity between variable and objective space. Computation requires knowing the global optimum; for the simulation-based problems we use the overall best found solution by all optimizers $\boldsymbol{x}^{(*)}$.

We use Sobol sequences of size $(n+2) \times 1000$ to acquire the samples for all problems except the solar and FFD problems. For those 4 problems we used the much smaller sample sizes using the same sequences as from the Morris analysis and hence FDC values should be ignored. We will conduct a larger sampling in future work for the solar and FFD problems.
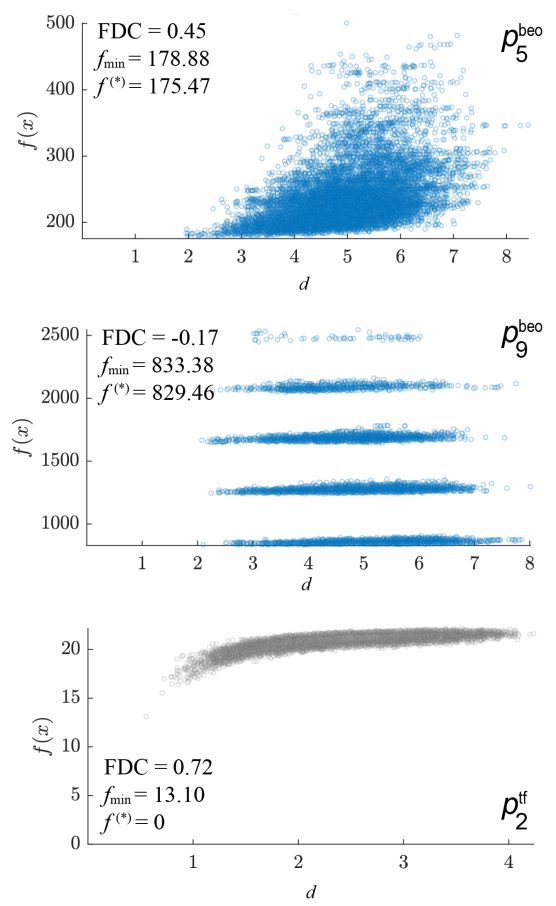

Figure 1: Fitness distance correlation plots for $p_{5}^{\text {beo }}$ (top), $p_{9}^{\mathrm{beo}}$ (middle) and the Ackley test function $p_{2}^{\mathrm{tf}}$ (bottom).

As an example, Fig. 1 shows FDC plots for $p_{5}^{\text {beo }}, p_{9}^{\text {beo }}$ and $p_{2}^{\mathrm{tf}}$. Different patterns emerge for the different problems: While $p_{2}^{\mathrm{tf}}$ shows a clear trend in approaching the global optimum $(\mathrm{FDC}=0.72), p_{5}^{\text {beo }}$ has a significantly higher spread $(\mathrm{FDC}=0.45)$. For $p_{2}^{\text {tf }}$ this might seem surprising given its known high degree of ruggedness, but it reveals a strong global structure with the unambiguous global optimum. The pattern for $p_{9}^{\text {beo }}$ looks different again with 5 stripes of elongated point clouds, revealing the impact of the penalty constraints (given in Waibel et al., 2019). It has a $\mathrm{FDC}=-0.17$, showing that this metric seems to face limitations with more complex (i.e. nonlinear) cost-distance distributions. Furthermore, from the result of $p_{2}^{\text {tf }}$ it is shown that FDC does not capture ruggedness. Still, the FDC values represent problem difficulty reasonably well, which will also be reflected in the benchmarking of optimizers shown later.

All FDC values are plotted as white squares in Fig. 3 for each problem respectively, summarized with other FLA metrics. Most test function problems have an FDC value of around 0.7 , the sphere function $p_{1}^{\mathrm{tf}}$ and $p_{10}^{\mathrm{tf}}$ are almost 1 indicating their relative easiness to solve. Only the constrained test function $p_{9}^{\text {tf }}$ has an FDC of around 0.5 , making it similar to many BEO problems. $p_{8}^{\text {beo }}$ to $p_{10}^{\text {beo }}$ have an FDC value near zero (we round negative FDC to 0 for simplification), indicating higher problem difficulty in these cases.

\section{Autocorrelation}

The autocorrelation of a timeseries is obtained by correlating it with a delayed copy of itself, where the number of steps of the delay is referred to as "lag". By computing the autocorrelation of a random walk an idea of the "ruggedness" of a landscape can be obtained and it therefore can serve as an indicator of problem difficulty (Pitzer and Affenzeller, 2012). High autocorrelation of a random walk indicates that if one step yields an improvement of the cost value, the subsequent step will likely also do. We propose to compute the median of the autocorrelation factors (ACF) from lag 1 to $n+1$ (i.e. one simplex gradient $k$ ) to represent the degree of ruggedness of a problem, which we denote as $\rho$.

Fig. 2 shows the ACF for $p_{5}^{\text {beo }}, p_{11}^{\text {beo }}$ and $p_{2}^{\text {tf }}$ as an example. The highly rugged landscape of the Ackley function $p_{2}^{\text {tf }}$ can be recognized by its low autocorrelation even with lag 1. $p_{5}^{\text {beo }}$ on the other hand shows high ACF even for longer lags, which indicates that even farther steps in a random walk are connected. $p_{11}^{\text {beo }}$ has gradually decreasing ACF the longer the lag, meaning that there is only an autocorrelation in a random walk for immediately following steps. It should be noted that the computed ACF need to be interpreted in relation to the used step size of the random walks (we used $2 \%$ of the problem domain). Therefore, smaller step sizes might reveal a finer degree of local ruggedness.

Fig. 3 shows the median ACF of lag 1 to $n+1$, $\rho$, visualized as star markers for all problems respectively. The figure shows very high $\rho$ up to 1 for BEO problems 1 to 8,13 and 14, slightly lower $\rho$ for BEO problems 9 to 11 , and relatively small $\rho$ of 0.6 to 0.5 for BEO problems 12 and 15 . Both solar problems and $p_{1}^{\text {wnd }}$ also show relatively high $\rho$. $p_{2}^{\text {wnd }}$ is the simulation-based problem in our analysis with the lowest $\rho$ of around 0.3 . The $\rho$ values for the test functions are as can be expected from their known characteristics: The smooth problems Sphere and Rosenbrock $\left(p_{1}^{\mathrm{tf}}, p_{4}^{\mathrm{tf}}, p_{5}^{\mathrm{tf}}\right.$ and $\left.p_{8}^{\mathrm{tf}}\right)$ have very high 


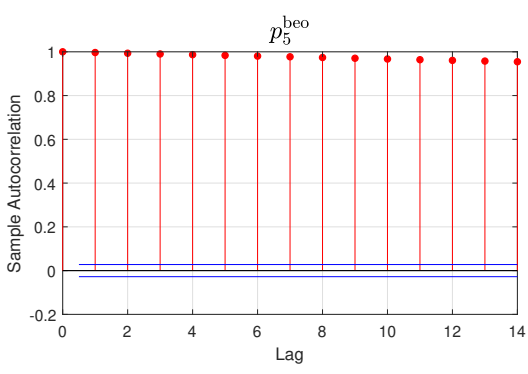

$p_{11}^{\text {beo }}$

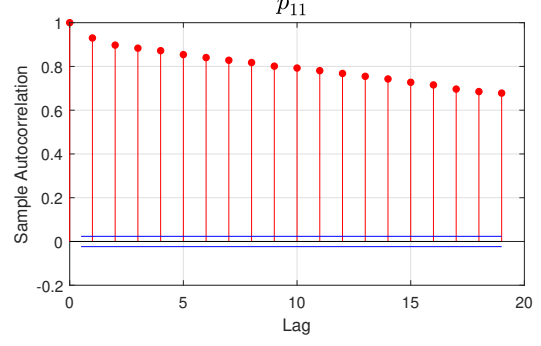

$p_{2}^{\mathrm{tff}}$

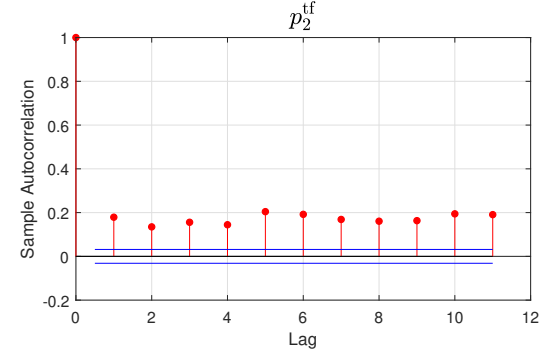

Figure 2: Autocorrelation factors for lags 1 to $n+1$ (=1k) for $p_{5}^{\text {beo }}$ (top), $p_{11}^{\text {beo }}$ (middle) and the Ackley test function $p_{2}^{\mathrm{tf}}$ (bottom).

$\rho$ of up to 1 ; the increase of problem dimension from 10 to 20 does not seem to have a significant impact on this metric. Furthermore, the rugged problems Ackley and Rastrigin $\left(p_{2}^{\mathrm{tf}}, p_{3}^{\mathrm{tf}}, p_{6}^{\mathrm{tf}}\right.$ and $\left.p_{7}^{\mathrm{tf}}\right)$ have as expected very low $\rho$ values. The constrained problem $p_{9}^{\text {tf }}$ shows relatively high $\rho$ similar to BEO problems 9 to 11 and both solar problems.

\section{Morris sensitivity analysis}

As an additional FLA step, we apply the Global Sensitivity Analysis (GSA) method of Morris, which was introduced in (Morris, 1991) and extended in (Campolongo et al., 2007). The aim is to identify the most important input parameters of a model with regards to output variations. Thus, it can be used for FLA in order to determine how many and which decision variables have the biggest impact on the cost function of an optimization problem.

As a measure of parameter importance, the Morris method calculates a metric $\mu_{i}^{*}$ per model decision variable with larger values indicating higher importance. To compute the Morris metric $r \cdot(n+1)$ model evaluations are required, where $r$ is a value with a minimum suggested value of 4-10 (Saltelli et al., 2004). Although $\mu_{i}^{*}$ is considered a primarily quantitative measure, it can give an indication of how much more significant a variable is compared to another one.
Fig. 3 shows the $\mu^{*}$ values for each variable and each problem respectively normalized by their total sum $\left(\left\|\mu_{i}^{*}\right\|=\mu_{i}^{*} / \sum \mu_{i}^{*}\right)$ to allow easy comparison of results between models. The figure furthermore groups decision variables into "geometry" (e.g. aspect ratios, window-to-wall-ratios, surface vertex positioning), "construction" (e.g. wall or window types, heavy or light weight, etc.), "energy system" (e.g. control set points, radiator sized, etc.) and "test function".

Besides the "families" that the most important variables belong to, it is also of interest whether for each problem only a few variables are significant or whether there is a more balanced contribution. To quantify this, for each problem we calculate the Coefficient of Variation $\left(c_{v}\right)$ of the normalized Morris metrics, which is defined as the ratio of the standard deviation to the mean of the $\left\|\mu_{i}^{*}\right\| . \quad c_{v}$ takes high values when few variables are important and many are unimportant, and low values when most variables exhibit similar importance levels.

In Fig. 3, for instance, $p_{1}^{\text {beo }}$ to $p_{3}^{\text {beo }}$ have low $c_{v}$ values, since three out of the four parameters are similarly important. Conversely in $p_{7}^{\text {beo }}$ and $p_{14}^{\text {beo }}$, the $c_{v}$ metric is higher since one variable is highly important. The most striking finding is that for almost all test function problems, the $c_{v}$ metric has a value of almost 0 , while for most of the BEO and solar problems the value is higher. This indicates that there are some "structural" differences between the test functions and the simulation-based problems, since in the latter most commonly just a few variables are primarily responsible for the variations of the cost function of interest.

\section{Benchmarking optimization algorithms}

In this section we compare the performance of many global black-box optimization (BBO) algorithms in solving the simulation-based problems and mathematical test functions, to investigate the hypothesis that there is no single algorithm that outperforms all others on the complete problem set. Furthermore, we are interested in finding performance differences between algorithms depending on the problem type, i.e. are some algorithms particularly good at test functions and others at building energy optimization (BEO) problems? We conduct 20 optimization runs per algorithm for each BEO problem and 5 for each solar, FFD and test function problem. We present the performance comparison using normalized boxplots, convergence graphs and a ranking matrix. For the boxplots and convergence graphs we show results separately for the BEO problems and the test functions and omit the solar and wind problems ${ }^{5}$. However, all

\footnotetext{
${ }^{5}$ We are trying to find whether there is a noticeable difference in performance of an algorithm for a certain set of problems (BEO or test functions), and we would need to include more solar and FFD problems to have a reasonable test set.
} 


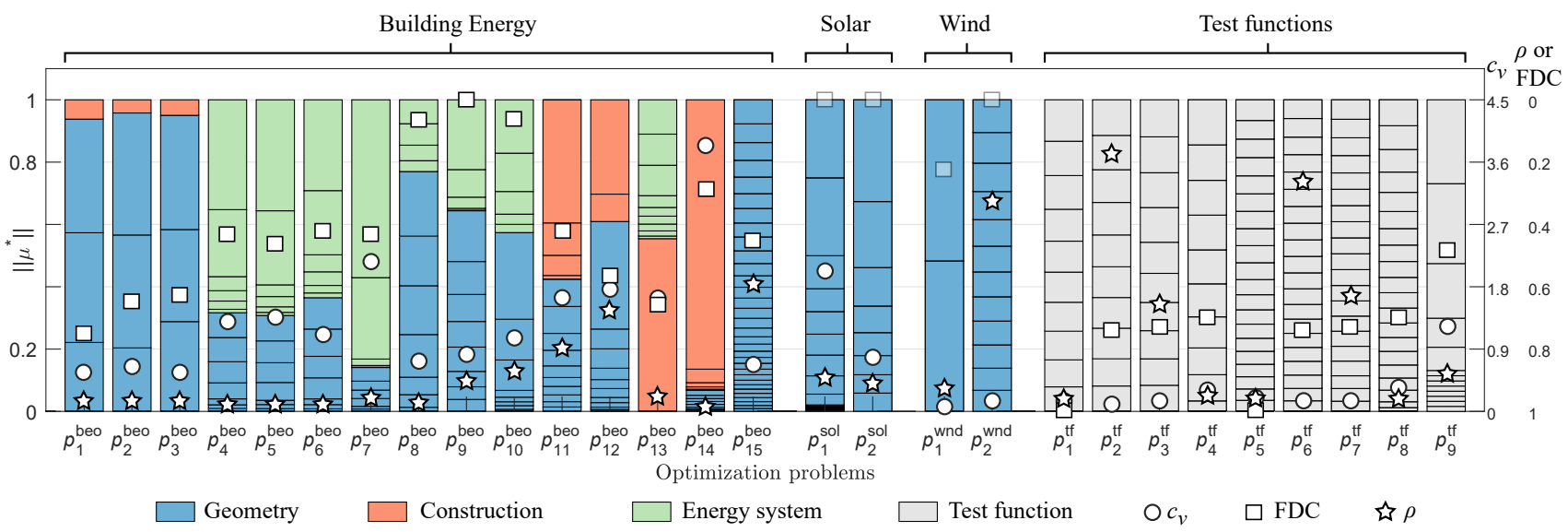

Figure 3: For each problem respectively: normalized Morris indices $\left\|\mu^{*}\right\|$; coefficient of variation of $\left\|\mu^{*}\right\|$, $c_{v}$; fitness distance correlation FDC; Autorcorrelation factor $\rho$. Larger values for $c_{v}$ indicate higher problem difficulty, vice versa for FDC and $\rho$.

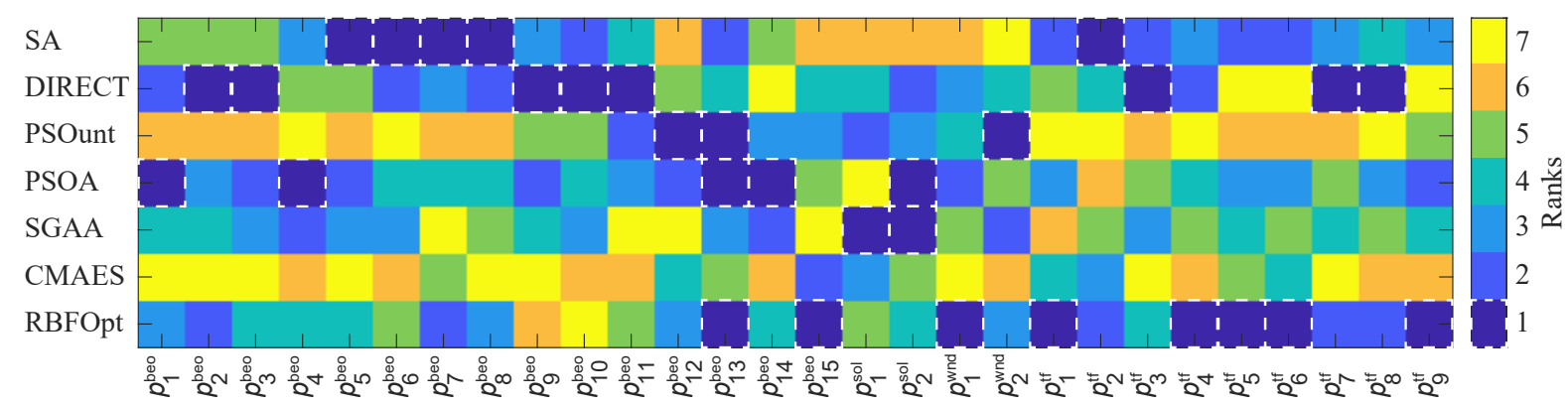

Figure 4: Ranked performance of all optimizers (i.e. median optimal solutions found at $k=30$ ) for each problem respectively, with rank 1 being best.

problems are included in the ranking matrix.

\section{Algorithms considered}

We include the following algorithms in our benchmark: RBFOpt ${ }^{6}$ (Costa and Nannicini, 2018) and CMA-ES $^{7}$ (Hansen et al., 2003) implemented in Rhino with Opossum ${ }^{8}$; the DIRECT (Jones et al., 1993) implementation from NLOpt ${ }^{9}$ coupled to Rhino with Goat ${ }^{10}$; Simulated Annealing (SA) (Kirkpatrick, 1984) which is installed by default in Rhino; and a simple Genetic Algorithm (SGAA) (Goldberg, 1989) and a Particle Swarm Algorithm (PSOA) (Eberhart and Kennedy, 1995) with tuned algorithmic parameters (Waibel et al., 2019) ${ }^{11}$, as well as an untuned version of the PSO, PSOunt from the open-source library BB-O ${ }^{12}$. The same algorithms have been used in Waibel et al. (2019), which lists the algorithm parameters. Default parameters have been used for all

\footnotetext{
${ }^{6}$ https://github.com/coin-or/rbfopt

${ }^{7}$ https://pypi.org/project/cma/

${ }^{8}$ https://aal.sutd.edu.sg/opossum/

${ }^{9}$ http: //ab-initio.mit.edu/nlopt

${ }^{10}$ http://www.rechenraum.com/goat/

${ }^{11}$ It should be noted that the tuning for SGAA and PSOA was applied using an evaluation budget of $k=100$ and therefore might not yield good results for the much shorter $k=30$ used in this paper.

12 https : //github.com/christophwaibel/BB-0
}

algorithms other than SGAA and PSOA.

\section{Results}

Fig. 4 shows the ranked performance of all algorithms per problem, with rank 1 being best. Performance is defined as the median cost value achieved by all optimization runs of an algorithm per problem. The figure demonstrates that there is no single algorithm that outperforms all others on the complete set of problems. SA performs very well on many BEO and test function problems, but ranks worse for $p_{15}^{\text {beo }}$ and all solar and FFD problems. DIRECT performs well on many problems as well, however shows the worst performance for $p_{14}^{\mathrm{beo}}, p_{5}^{\mathrm{tf}}, p_{6}^{\mathrm{tf}}$ and $p_{9}^{\mathrm{tf}}$. For RBFOpt it is interesting to note that it performs best for many test functions. Similar observations can be made for all algorithms: they only show good performance on a subset of the problem set. There is no single algorithm dominating all others on all problems, or under all conditions (low versus high $k$, other performance metrics, etc.).

Fig. 5 shows normalized ${ }^{13}$ boxplots and convergence graphs of the cost values. The convergence graphs

\footnotetext{
${ }^{13}$ The normalization of the cost values is conducted with the worst and best optimal solution found at $k=30$ by all algorithms.
} 
Optimal cost values found at $k=30$ for $p^{b e o}$

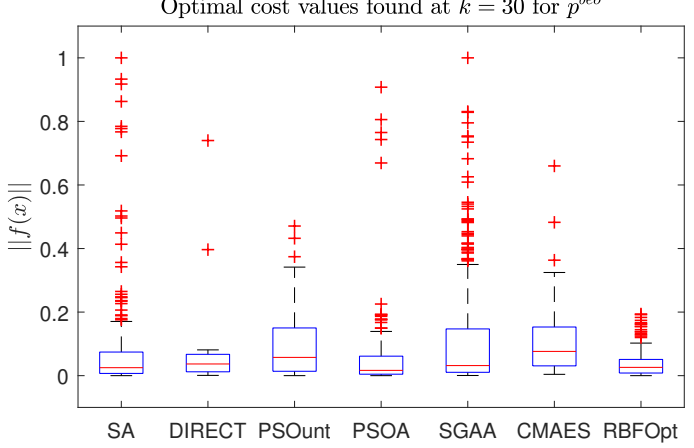

Optimal cost values found at $k=30$ for $p^{t f}$

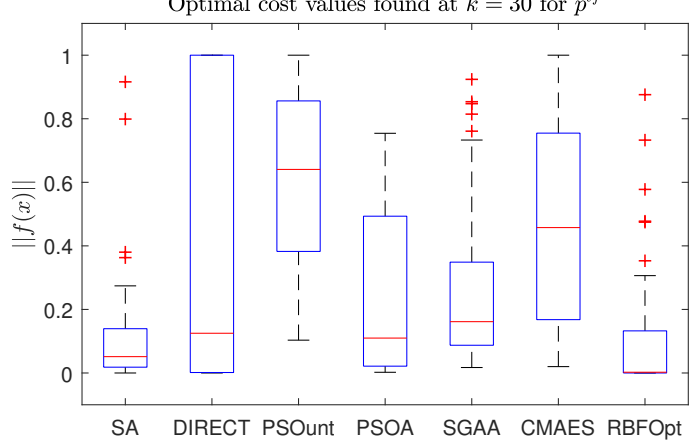

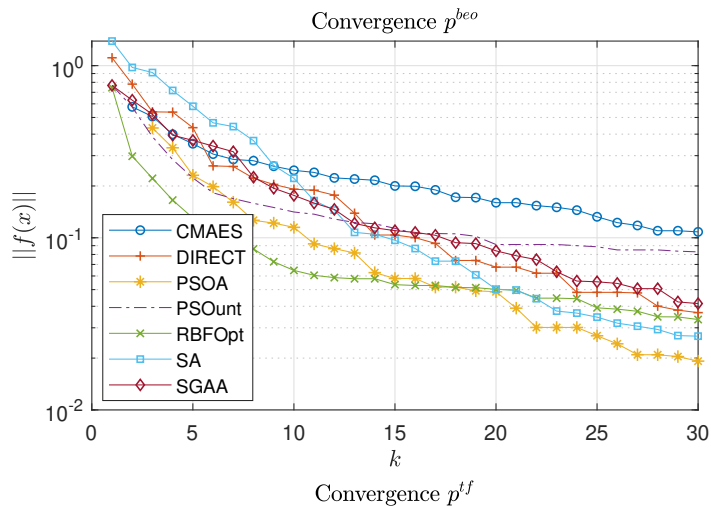

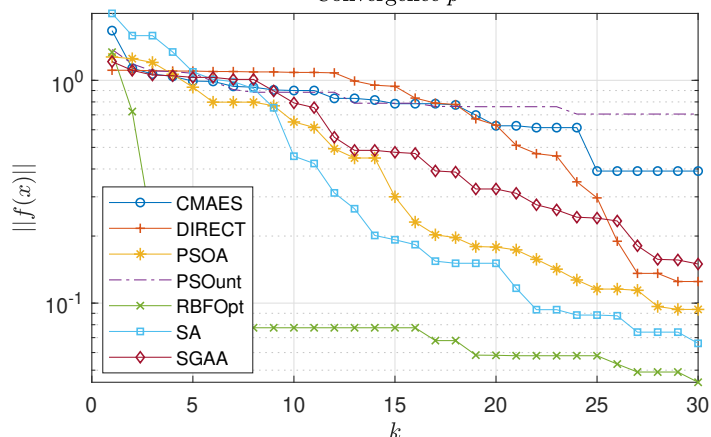

Figure 5: (Left) Boxplots of normalized cost values at $k=30$ of all problems and runs. (Right) Combined normalized median cost values of all runs and problems as a function of simplex gradient $k$. Top row shows results for $\boldsymbol{p}^{\text {beo }}$ only and bottom row for $\boldsymbol{p}^{\mathrm{tf}}$ only.

show the median cost values of all runs per algorithm as a function of $k$, the boxplots are for $k=30$ only. As mentioned earlier, Fig. 5 separates results for the set of $\mathrm{BEO}$ and the set of test function problems and omits the solar and FFD problems. For the BEO problems the boxplots show close median values up to $\|f(\boldsymbol{x})\|=0.1$ achieved by all algorithms, with $\mathrm{SA}$, PSOA, SGAA and RBFOpt having the best median values. However, RBFOpt shows the best (i.e. smallest) spread demonstrating its general robustness. DIRECT and PSOA also have a low spread albeit with some outliers. Comparing the boxplots of the BEO problem set to those of the test function set shows that SA and RBFOpt are the best regarding median and spread. DIRECT, PSOA and SGAA also have reasonably low median values but significantly higher spreads.

Most striking in the convergence graphs is how fast RBFOpt achieves low cost values for the test function set and maintains its unassailable lead until the final evaluation count $k=30$. However for the BEO problem set, this algorithm loses first place to PSOA at around $k=15$ and is overtaken by SA at $k=20$ and SGAA and DIRECT coming close at $k=30$.

\section{Discussion}

\section{Synthetic BEO test functions?}

It appears that benchmarks from the heuristic optimization community often try to achieve a high generality, i.e. that an algorithm is good at as many different problems as possible. Burke et al. (2009) propose that an algorithm should perform well on as many different problems as possible, rather than becoming good at a certain type of problem. However, we argue that by doing so we lose the potential to design or calibrate an algorithm such that it becomes especially effective and efficient in a certain problem domain - e.g. building simulation.

Our fitness landscape analysis (FLA) results indicate that there are distinct features present in one problem domain not present in another (e.g. significantly lower FDC values and significantly higher $\rho$ and $c_{v}$ in BEO than in test functions). The next step would be to discriminate simulation-based problems in the domain of building simulation from other domains and especially from mathematical test functions. This would incentivize the development of specialized building-simulation optimization algorithms with superior performance in this domain. This concept also coincides with the "No Free Lunch" theorem in optimization, which can be interpreted as stating that if we improve the performance of an algorithm in one domain, we decrease its performance in another (Wolpert and Macready, 1997).

One way of systematically discriminating problems would be to use clustering techniques on a large problem set according to a number of FLA metrics (such as FDC, $\rho$ and $c_{v}$ ). This would show us (dis)similarities amongst problems and group them together meaningfully. However, open questions remain: which features are significant and most useful 
for clustering (i.e. information gain)? Are the presence of constraints, discrete variables, or the type of variable (geometry, construction, energy system etc.), or even the type of simulator (EnergyPlus, FFD, solar model) meaningful features for discrimination? And how large should the problem set be in order to conduct a sufficiently generalizable clustering?

Such questions should be addressed in future work, but certainly more real-world building design problems should be included in an analysis, along with more FLA metrics. The problems included in this paper do not provide a large enough data set for drawing sufficiently confident conclusions for creating problem clusters. The FLA metrics, while giving hints on certain structural differences between simulation-based problems and mathematical test functions, are not entirely unambiguous.

Given a larger set of representative buildingsimulation problems, a thorough FLA and clustering could provide us with the information to create synthetic training functions that well-represent BEO problems. These could be used when designing or tuning BBO algorithms instead of the generic mathematical test functions currently used.

\section{Building energy optimization competition}

On a final note, based on the above conclusions we propose a building energy optimization competition, similar to those for general optimizers (GECCO, CEC, COCO, BBCOMP, etc.). This would provide a standard benchmark for testing and developing optimization algorithms suitable for building energy problems. It would incentivize improvements to algorithms specifically aimed at building simulations. Subcategories could include CFD or FFD, building energy, solar, energy systems and network design problems as well as many others. The competition could be part of the bi-yearly IBPSA conferences, and could also run on a rolling basis online. Results would span various metrics, allowing the best algorithms to be listed for each problem type, metric etc.

The competition could be structured in various ways. An online platform could conduct the evaluation calls, thus tracking the calls per competitor. Black-box executable files could be provided that also log evaluation calls. Synthetic training functions could be fitted using machine learning, avoiding the need for real simulators to be executed every time. These could be based on a very dense sampling of the problem space, or even brute-force evaluation of all solutions if the problem is discrete. Problems could also be randomized, to avoid algorithms being over-tuned to the particular problem instances used in the competition. Ideally the problems, which could change periodically, would remain entirely unknown to the participants. Details of problem characteristics could be provided, or left to the competitors to discover.
As a starting point, we provide the open-source BEO problem testbed $^{14}$ from Waibel et al. (2019). This is already more extensive than all previous building energy optimization benchmarks. The benchmark can be expanded to include other published problems. The next step will be to collate user-submitted algorithms and associated results ${ }^{15}$ on a website, allowing visitors to see the best algorithms across the problems and metrics used in this paper.

\section{Summary and conclusion}

This paper conducted a fitness landscape analysis (FLA) of a set of simulation-based building energy optimization (BEO), solar and air flow problems and compared their problem structures to those of common mathematical test functions. The FLA revealed that BEO problems are significantly different to test functions for a number of FLA metrics, such as fitness distance correlation, coefficient of variation, and autocorrelation factors.

We compared the performance of a number of global black-box optimization (BBO) algorithms and found that there is no single algorithm that outperforms all others for the complete problem set. The benchmark and the FLA together indicate that it is possible to discriminate simulation-based building design problems from other domains and specifically from mathematical test functions. Discipline-specific problem characteristics should therefore be exploited in the improvement of optimization algorithms, rather than advocating a higher generalization such as an algorithm that performs well overall but sacrifices performance in the domain of building optimization.

To facilitate developments in BEO we provide an open-source test bed of EnergyPlus problems, as published in Waibel et al. (2019). Furthermore, we propose regular optimization competitions, providing the framework for researchers to benchmark their own BBO algorithms. This would support the development of better algorithms and ultimately facilitate more effective use of optimization in real-world building design problems.

\section{Acknowledgments}

This research has been financially supported by the Swiss Commission for Technology and Innovation within SCCER FEEB\&D (CTI 1155002539) and by the Swiss Competence Center for Energy and Mobility project SECURE (CCEM 914).

\section{References}

Audet, C. and W. Hare (2017). Derivative-Free and Blackbox Optimization. Cham: Springer Series in

\footnotetext{
${ }^{14}$ https ://github.com/christophwaibel/BEOBenchmark

${ }^{15}$ An honesty-based approach is reasonable, since results are reproducible.
} 
Operations Research and Financial Engineering, Springer International Publishing AG.

Burke, E. K., T. Curtois, G. Kendall, M. Hyde, G. Ochoa, and J. A. Vazquez-Rodriguez (2009). Towards the decathlon challenge of search heuristics. Proceedings of the 11th annual conference companion on Genetic and evolutionary computation conference - GECCO '09, 2205.

Campolongo, F., J. Cariboni, and A. Saltelli (2007). An effective screening design for sensitivity analysis of large models. Environmental Modelling and Software 22(10), 1509-1518.

Costa, A. and G. Nannicini (2018). RBFOpt : an open-source library for black-box optimization with costly function evaluations. Mathematical Programming Computation 10(4), 597-629.

Crawley, D. B., L. K. Lawrie, C. O. Pedersen, and F. C. Winkelmann (2000). EnergyPlus: Energy Simulation Program. ASHRAE Journal 42, 49-56.

Djuric, N., V. Novakovic, J. Holst, and Z. Mitrovic (2007). Optimization of energy consumption in buildings with hydronic heating systems considering thermal comfort by use of computer-based tools. Energy and Buildings 39(4), 471-477.

Eberhart, R. and J. Kennedy (1995). A new optimizer using particle swarm theory. Proceedings of the Sixth International Symposium on Micro Machine and Human Science, 39-43.

Evins, R. (2013). A review of computational optimisation methods applied to sustainable building design. Renewable and Sustainable Energy Reviews 22, 230-245.

Goldberg, D. E. (1989). Genetic Algorithms in Search, Optimization, and Machine Learning (1st ed.). Addison-Wesley Publishing Company Inc.

Hamdy, M., A.-T. Nguyen, and J. L. Hensen (2016). A performance comparison of multi-objective optimization algorithms for solving nearly-zero-energybuilding design problems. Energy and Buildings 121, 57-71.

Hansen, N., S. D. Müller, and P. Koumoutsakos (2003). Reducing the Time Complexity of the Derandomized Evolution Strategy with Covariance Matrix Adaptation (CMA-ES). Evolutionary Computation 11(1), 1-18.

Jones, D. R., C. D. Perttunen, and B. E. Stuckman (1993). Lipschitzian optimization without the Lipschitz constant. Journal of Optimization Theory and Applications 79(1), 157-181.
Jones, T. and S. Forrest (1995). Fitness Distance Correlation as a Measure of Problem Difficulty for Genetic Algorithms. In Proceedings of the 6th International Conference on Genetic Algorithms, Volume 95, pp. 184-192.

Kämpf, J. H. and D. Robinson (2010). Optimisation of building form for solar energy utilisation using constrained evolutionary algorithms. Energy and Buildings 42(6), 807-814.

Kämpf, J. H., M. Wetter, and D. Robinson (2010). A comparison of global optimization algorithms with standard benchmark functions and real-world applications using EnergyPlus. Journal of Building Performance Simulation 3(2), 103-120.

Kirkpatrick, S. (1984). Optimization by simulated annealing: Quantitative studies. Journal of statistical physics 34, 975-986.

Morris, M. D. (1991). Factorial Sampling Plans for Preliminary Computational Experiments. Technometrics 33(2), 161-174.

Nguyen, A. T. and S. Reiter (2014). Passive designs and strategies for low-cost housing using simulation-based optimization and different thermal comfort criteria. Journal of Building Performance Simulation 7(1), 68-81.

Nguyen, A.-T., S. Reiter, and P. Rigo (2014). A review on simulation-based optimization methods applied to building performance analysis. Applied Energy 113, 1043-1058.

Pitzer, E. and M. Affenzeller (2012). A Comprehensive Survey on Fitness Landscape Analysis. Recent Advances in Intelligent Engineering Systems, 161191.

Ramallo-González, A. P. and D. A. Coley (2014). Using self-adaptive optimisation methods to perform sequential optimisation for low-energy building design. Energy and Buildings 81 (September 2012), 18-29.

Rios, L. M. and N. V. Sahinidis (2013). Derivativefree optimization: A review of algorithms and comparison of software implementations. Journal of Global Optimization 56(3), 1247-1293.

Saltelli, A., S. Tarantola, F. Campolongo, and M. Ratto (2004). Sensitivity analysis in practice: a guide to assessing scientific models. John Wiley \& Sons.

Waibel, C., L. Bystricky, A. Kubilay, R. Evins, and J. Carmeliet (2017). Validation of Grasshopperbased Fast Fluid Dynamics for Air Flow around Buildings in Early Design Stage. In Building Simulation 2017, August 7-9, San Francisco, California, $U S A$. 
Waibel, C., R. Evins, and J. Carmeliet (2016). Holistic Optimization of Urban Morphology and District Energy Systems. In G. Habert and A. Schlueter (Eds), Systems Thinking in the Built Environment. Sustainable Built Environment (SBE) Regional Conference Zurich, June 15th 17th, pp. 7076. vdf Hochschulverlag AG ETH.

Waibel, C., R. Evins, and J. Carmeliet (2017). Efficient time-resolved 3D solar potential modelling. Solar Energy 158(October), 960-976.

Waibel, C., R. Evins, and J. Carmeliet (2019). Clustering and Ranking Based Methods for Selecting Tuned Search Heuristic Parameters. In IEEE Congress on Evolutionary Computation, Wellington, New Zealand, pp. 2932-2941.

Waibel, C., T. Wortmann, R. Evins, and J. Carmeliet (2019). Building Energy Optimization: An Extensive Benchmark of Global Search Algorithms. Energy and Buildings 18\%, 218-240.

Wetter, M. and J. Wright (2004). A comparison of deterministic and probabilistic optimization algorithms for nonsmooth simulation-based optimization. Building and Environment 39(8), 989-999.

Wilkinson, S. and S. Hanna (2014). Approximating Urban Wind Interference. In Symposium on Simulation for Architecture and Urban Design.

Wolpert, D. H. and W. G. Macready (1997). No free lunch theorems for optimization. IEEE Transactions on Evolutionary Computation 1(1), 67-82.

Wortmann, T., C. Waibel, G. Nannicini, R. Evins, T. Schroepfer, and J. Carmeliet (2017). Are Genetic Algorithms Really the Best Choice for Building Energy Optimization ? In Proceedings of the Symposium on Simulation for Architecture \& Urban Design (SimAud), Toronto, Canada, pp. 5158.

Yi, Y. K. and H. Kim (2015). Agent-based geometry optimization with Genetic Algorithm (GA) for tall apartment's solar right. Solar Energy 113, 236-250. 